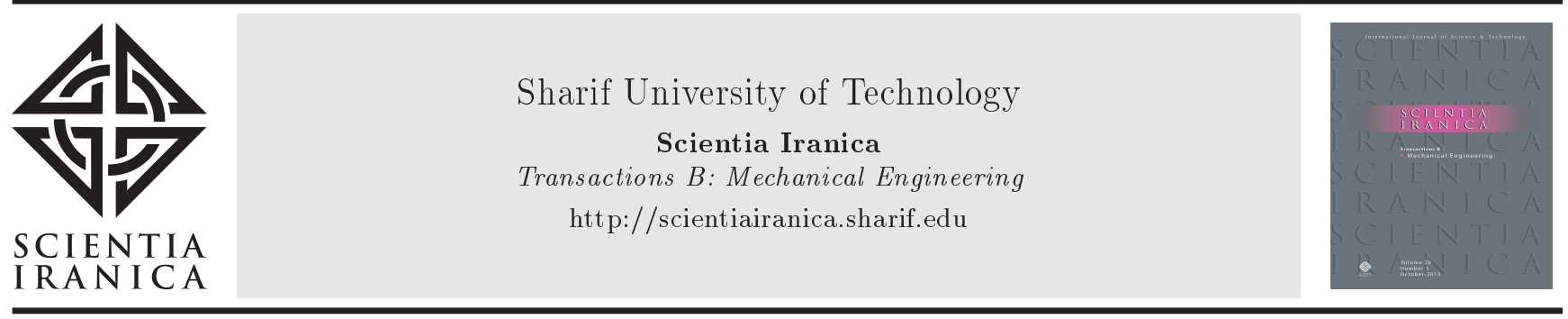

\title{
Role of movement of walls with time-dependent velocity on flow and mixed convection in vertical cylindrical annulus with suction/injection
}

\author{
A. Shakiba and A.B. Rahimi* \\ Department of Mechanical Engineering, Ferdowsi University of Mashhad, Mashhad, P.O. Box 917r5-1111, Iran.
}

Received 6 January 2020; received in revised form 6 June 2020; accepted 3 August 2020

\author{
KEYWORDS \\ Mixed convection; \\ Vertical annulus; \\ Time-dependent \\ moving walls; \\ Transpiration; \\ Exact solution.
}

\begin{abstract}
The unsteady mixed convection flow within a vertical concentric cylindrical annulus with time-dependent moving walls is investigated. Fluid is suctioned/injected through the cylinder walls of the annulus. The effects of wall movement on flow and heat transfer characteristics inside the cylindrical annulus are sought. An exact solution of Navier-Stokes and energy equations is obtained in this problem, for the first time. Here, the heat transfer occurs from the walls of the hot cylinder with constant temperature to the moving fluid with a cooling role. It is interesting to note that the results indicate that the time-dependency of cylinder wall movements has no effect on the temperature profile. The results also indicate that usage of an inverse of time for velocity movement causes a significant increase in velocity, stress tensor, and Nusselt number in the vicinity of the moving wall. Also, increasing the mixed convection and suction/injection parameters increases the Nusselt number and decreases the stress tensor on the inner and outer cylinders, respectively; no matter what velocity function is chosen for the moving wall. Therefore, using the velocity function of the wall movement and the change in other nondimensional governing parameters, flow and heat transfer can be controlled.
\end{abstract}

(C) 2021 Sharif University of Technology. All rights reserved.

\section{Introduction}

The study of a viscous fluid flow in the space between vertical concentric cylinders whose cylindrical walls move in the axial direction with time-dependent velocity is of great importance theoretically and practically, and it is the subject of extensive research in various parts of engineering. Applications of an annulus include cylindrical tanks design, heat exchangers, new cooling systems in advanced nuclear reactors, thermal energy storage cells, cooling of electronic devices, solar energy collectors, drilling, the elimination of atheroscle-

*. Corresponding author.

E-mail address: rahimiab@um.ac.ir (A.B. Rahimi) rosis, the fault detection of oil pipelines and so on. In laboratory work, Wu et al. [1] surveyed the mixed convective flow of water inside a vertical annulus. The results revealed that the effect of buoyancy force is weaker than the other states when the constant flux boundary condition is considered on the inner wall and adiabatic on the outer wall. In an analytical work, Joshi [2] studied the free convective flow within an annulus channel. The results demonstrated that Nusselt number and flow rate are dependent on the annulus gap and the ratio of dimensionless temperature. Chen et al. [3] examined the flow and heat transfer in a vertical annulus numerically. The obtained results showed that Nusselt number depends on the radial ratio. Iannello et al. [4] studied the laminar flow and mixed convective heat transfer in an annulus section at the constant temperature of the walls. The results of this research 
indicated the dependency of the friction coefficient and Nusselt number on the wall on the mixed convective parameter. Malvandi et al. [5] concluded that the buoyancy force has a negative effect on the efficiency of the system by examining the mixed convective flow of nanofluid in the vertical annulus and in the constant heat flux boundary conditions on the walls. Avcr and Aydın [6] analytically investigated the mixed convective flow within an annulus in a fully developed state. They revealed that the enhancement of mixed convective parameters increases heat transfer. Jha et al. [7] investigated the behavior of mixed convective flow in a micro-annulus for a viscous incompressible fluid. They came to the conclusion that as the suction and fluid injection into the walls increased, the temperature and velocity of the fluid heightened. Jha et al. [8] also studied the effects of the suction/injection of the fluid in a vertical micro-porous annulus. They concluded that when the fluid is injected, the friction is reduced at the inner cylinder surface. It should be noted that these results are reversed for the outer cylinder. In an experimental-numerical work, Husain and Siddiqui [9] examined transient natural convection flow within an annulus. The results indicated that a drop in the transient period appeared with the enhancement of heat input. Moreover, they showed that annulus height causes the gradual augmentation of the transient period. In another study, Husain et al. [10] concluded that the Nusselt number increases and decreases with the enhancement of radius ratio and aspect ratio, respectively, by investigation of geometrical parameters affecting the natural convection flow in a vertical annulus. Some other relative studies have also been conducted in the field of exploring flow and heat transfer characteristics in annulus geometry [11-13].

Fluid flow and heat transfer within concentric cylinders with movable walls have always fascinated researchers owing to their usage in manufacturing and the cooling processes of thermal equipment. Moreover, forecasting the distribution of transient state temperature and heat transfer rate from the initial state to a steady state is of great importance in some engineering issues. It needs to be stated that the application of such cases are as follows: Extrusion processes in productive industries, polymer pipe coating, simulation of a passing train through a long tunnel, drilling operations, cooling processes such as hot rolling, nuclear reactors and nuclear fuel channels [14-16]. Shigechi and Lee [17] investigated the fluid flow and heat transfer of an annulus with a movable wall. They discovered that the friction decreased by increasing the velocity of the movable wall and the Nusselt number changes are strongly dependent on the thermal boundary conditions. In an analytical work, Chamkha [18] surveyed the flow and heat transfer of the fluid in a vertical moving plate in an unsteady state and with the existence of a magnetic field. The results represented that Nusselt number and the skin friction coefficient declined by augmenting the thermal absorption coefficient. In a numerical work, Abedini and Rahimi [19] scrutinized the mixed convective heat transfer in an annulus consisting of two horizontal and rotating concentric cylinders. They investigated the effect of different rotation functions as time-dependent boundary conditions on inner and outer cylinders. Saleh and Rahimi [20] inspected the unsteady flow and heat transfer around the stagnation point of an infinite and moving cylinder with time-dependent axial velocity and uniform normal transpiration as an exact solution. In a numerical work, Jha et al. [21] examined the natural convection flow in a transient state in a vertical porous annulus. Temperature and velocity are independent of Prandtl number in a steady state when there is no suction/injection, but are dependent on Prandtl number if the suction/injection exists. Jha et al. [22] dealt with the fully developed steady/unsteady flow and the natural convection flow in a vertical annulus. They found that with the enhancement of dimensionless time, the dimensionless temperature and velocity increase until they reach a steady state. Through acquiring the results of an exact solution, Shakiba and Rahimi [23] studied the fluid flow and heat transfer features within a vertical cylindrical annulus by considering the effects of radial magnetic field and suction/injection. Busedra and Tavoularis [24] investigated natural convection flow by considering a vertical annular channel. Their numerical solution results indicated that the heat transfer rate for the concentric annular channel was higher than the eccentric annular channel under the same thermal conditions. Moreover, the mass flow rate in eccentric channel mode is higher than in the concentric mode. Hekmat and Ziarati [25] studied the effect of volume fraction changes and magnetic field gradients on Ferrofluid mixed convection flow within a vertical annulus. The numerical results revealed that when the magnetic field gradient is negative, the skin friction coefficient increases and decreases respectively on the inner and outer walls by enhancing the volume fraction and magnetic field gradient. Jha et al. [26] investigated the natural convection flow in a vertical annulus whose walls are subject to periodic heat flux, which is a function of time. The analytical results showed that by augmenting the Strouhal number and Prandtl number, the heat transfer rate increased. Jha et al. [27] surveyed the natural convection flow within a micro-channel. Through obtaining analytical solution results, they concluded that the increase of Hall current heightens the fluid velocity in both primary and secondary directions of flow. Abbas et al. [28] studied the natural convection flow of Ferrofluid in a vertical micro cylindrical tube. The effects of a radial magnetic 
field, as well as the effects of suction/injection, were also considered in this study. The results of the analytical solution showed that fluid velocity decreased with enhancement of the injection parameter, and fluid velocity increased with augmentation of the suction parameter. Furthermore, the opposite of these results is confirmed for temperature distribution.

Although much research has been conducted in this area, the mixed convective flow of the fluid in an annulus, whose walls move at a time-dependent velocity, has not been studied so far. Accordingly, the fluid flow passing through an annulus and its heat transfer in a transient state is scrutinized in this article by selecting time-dependent functions for the cylinder wall.

In this study, the effect of cylinder walls that move in an axial direction with constant velocity, on various parameters, was investigated. The results revealed that the increase of the mixed convective parameter and the suction/injection parameter without the presence of a magnetic field led to the growth of shear stress and heat transfer rate on the inner cylinder wall. Moreover, the movement of each of the inner and outer cylinders gives rise to the highest and lowest heat transfer rate, respectively, on the inner cylinder wall.

\section{Problem statement}

In the present study, the behavior of the flow and heat transfer of the fluid within concentric cylinders is examined when the walls of the cylinders move in the axial direction with time-dependent velocity. According to Figure 1, a cylindrical coordinate system $(r, \theta, z)$ is used, where the geometry of the annulus consists of two vertical and concentric cylinders with a circular section where the $z$ axis is along with the annulus axis and the $r$ axis is perpendicular to it. It should be stated that the values of radius and temperature are considered $a$ and $T_{1}$, respectively, for the inner cylinder, and these values are assumed $b$ and $T_{0}$, respectively, for the outer cylinder. In addition, the temperature of the inner cylinder is higher than the outer cylinder. The walls of inner and outer cylinders are considered perforated and fluid is suctioned/injected from/into them. To solve this problem, the equations of continuity, momentum and energy need to be solved, along with the boundary conditions in a transient state. By doing so and changing the parameters governing the problem, the fluid flow can be controlled and the heat transfer of the system can be increased.

As a matter of note, the relevant results will be presented in this article through obtaining the dimensionless equations related to the flow and heat transfer and solving them. The present study is a fundamental step for better understanding of the effects of wall motion, along with the suction/injection of the fluid, on improving the efficiency of heat exchangers. If this

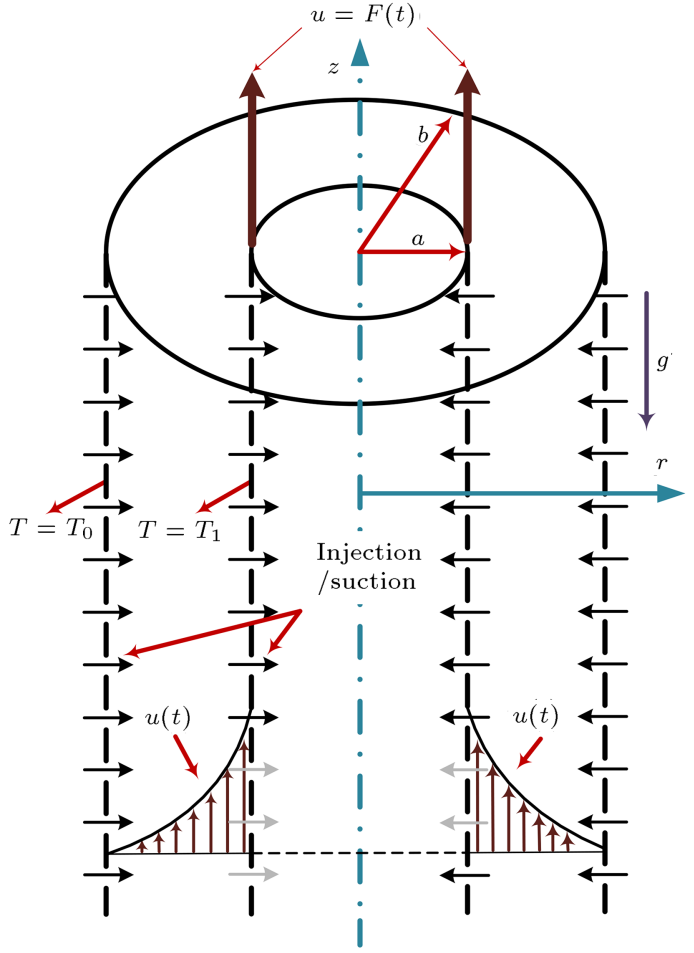

Figure 1. Problem geometry.

method leads to the dramatic increase of heat transfer in heat exchangers, its industrial use will satisfy a basic need in the industry, which is an increase in the heat transfer of heat exchangers while their volume reduces.

\section{Governing equations and boundary conditions}

The fluid flow is taken into account as laminar, transient, fully developed, Newtonian and incompressible. By exerting a no slip condition to the walls and employing a Boussinesq approximation, the conservation equations, which include the equations of continuity, momentum and energy, are written according to Eqs. (1)(3). The viscous dissipation and axial conduction of the fluid and wall are foregone in writing these equations. With regard to annulus length, which is infinite, all the physical parameters of the problem are a function of $r$, except the pressure, which is a function of $z$.

Continuity equation:

$$
\frac{1}{r} \frac{\partial}{\partial r}\left(r V_{r}\right)=0 \text {. }
$$

Momentum equation:

$$
\begin{aligned}
\frac{\partial V_{z}}{\partial t} & +V_{r} \frac{\partial V_{z}}{\partial r}=-\frac{1}{\rho} \frac{\partial p}{\partial z}+\frac{\nu}{r} \frac{\partial}{\partial r}\left(r \frac{\partial V_{z}}{\partial r}\right) \\
& +g \beta\left(T-T_{0}\right) .
\end{aligned}
$$

Energy equation: 


$$
\frac{\partial T}{\partial t}+V_{r} \frac{\partial T}{\partial r}=\frac{\alpha_{T}}{r} \frac{\partial}{\partial r}\left(r \frac{\partial T}{\partial r}\right) .
$$

In Eqs. (1) to (3), $\alpha_{T}, g$ and $\beta$ are thermal diffusivity, gravity and the thermal expansion coefficient of the fluid, respectively.

Boundary conditions are also defined as in Eq. (4). With regard to the boundary conditions, the cylinder walls were initially stationary and the temperature of all parts was constant and equal to $T_{0}$. Then, the wall of the inner cylinder is set at the temperature of $T_{1}$ at the moment of $(t>0)$, and it moves in the direction of the cylinder axis with the velocity of $F(t)$ which is time dependent.

$$
\begin{array}{ccc}
t=0 & T(r, 0)=T_{0} & V_{z}=(a, 0)=0 \\
& & V_{z}(b, 0)=0 \\
t>0 & T(a, t)=T_{1} & V_{z}(a, t)=F(t) \\
& T(b, t)=T_{0} & V_{z}(b, t)=0
\end{array}
$$

The velocity component in the radial direction can be obtained in the form of $V_{r}=\frac{-a V_{0}}{r}$ through integration of Eq. (1) and using the boundary condition of $V_{r}=$ $-V_{0}$ at $r=a$. In the following, the velocity component in the axial direction, $V_{z}$, will be displayed as $u$.

The dimensionless parameters related to the present problem are defined as in the following:

$$
\begin{aligned}
& \tau=\frac{t \nu}{a^{2}}, \quad R=\frac{r}{a}, \quad \lambda=\frac{b}{a}, \quad \theta=\frac{T-T_{0}}{T_{1}-T_{0}}, \\
& U=\frac{u}{u_{0}}, \quad S=\frac{V_{0} a}{\nu}, \quad \operatorname{Gr}=\frac{g \beta \Delta T D_{h}^{3}}{\nu^{2}}, \\
& \operatorname{Pr}=\frac{\nu}{\alpha_{T}}, \quad \operatorname{Re}=\frac{u_{0} D_{h}}{\nu}, \quad \eta=G r / \operatorname{Re} .
\end{aligned}
$$

In which $u_{0}, D_{h}, \nu, \alpha_{T}$, and $\Delta T$ are the reference velocity, hydraulic diameter, kinematic viscosity, thermal diffusivity and temperature difference, respectively, and they are defined according to:

$$
\begin{aligned}
& \mathrm{u}_{0}=\frac{-D_{h}^{2}}{\mu} \frac{d p}{d z}, \quad D_{h}=2(b-a), \quad \nu=\frac{\mu}{\rho}, \\
& \alpha_{T}=\frac{k}{\rho C_{p}}, \quad \Delta T=T_{1}-T_{0} .
\end{aligned}
$$

Regarding Figure 1 and Eq. (5), $S$ is defined as the suction/injection parameter and $S<0$ represents a situation in which the suction takes place in the direction of the annulus to the walls. $S>0$ indicates the opposite of the above situation.

The dimensionless format of conservation equations, including momentum and energy, along with the boundary conditions are expressed in terms of Eqs. (7)-(9).

The boundary conditions are:

$$
\begin{array}{ccc}
\tau=0 & \theta(R, 0)=0 & u(1,0)=0 \\
& & U(\lambda, 0)=0 \\
& \theta(1, \tau)=1 & U(1, \tau)=F(\tau) \\
\tau>0 & \theta(\lambda, \tau)=0 & U(\lambda, \tau)=0
\end{array}
$$

The momentum equation becomes:

$$
\begin{aligned}
\frac{\partial U(R, \tau)}{\partial \tau} & -\frac{S}{R} \frac{\partial}{\partial R} U(R, \tau)-\frac{1}{R} \frac{\partial}{\partial R}\left(R \frac{\partial}{\partial R} U(R, \tau)\right) \\
& -\frac{1}{4(\lambda-1)^{2}}[1+\eta \theta(R, \tau)]=0
\end{aligned}
$$

The energy equation is:

$$
\begin{aligned}
\frac{\partial \theta(R, \tau)}{\partial \tau} & -\frac{S}{R} \frac{d}{d R} \theta(R, \tau) \\
& =\frac{1}{\operatorname{Pr}} \frac{1}{R} \frac{d}{d R}\left(R \frac{d}{d R} \theta(R, \tau)\right) .
\end{aligned}
$$

Dimensional shear stress and Nusselt number on the walls of inner and outer cylinders are defined according to the following relations:

$$
\begin{aligned}
& \left.\sigma\right|_{@ \text { wall }}=\left.\frac{\partial U}{\partial R}\right|_{@ \text { wall }} \cdot \\
& \left.\mathrm{Nu}\right|_{R=1, \tau}=-2(\lambda-1) \frac{\left.\frac{\partial \theta}{\partial R}\right|_{R=1, \tau}}{\theta(1, \tau)-\theta_{b}}, \\
& \left.\mathrm{Nu}\right|_{R=\lambda, \tau}=2(\lambda-1) \frac{\left.\frac{\partial \theta}{\partial R}\right|_{R=\lambda, \tau}}{\theta(\lambda, \tau)-\theta_{b}} .
\end{aligned}
$$

In Eq. (11), $\theta_{b}$ is the dimensionless bulk temperature of the fluid and is defined according to the following:

$$
\theta_{b}=\frac{T_{b}-T_{0}}{T_{1}-T_{0}}=\frac{\int_{1}^{\lambda} R U(R, \tau) \theta(R, \tau) d R}{\int_{1}^{\lambda} R U(R, \tau) d R} .
$$

In the following, the solution results are presented.

\section{Validation}

To prove the accuracy of the results, the velocity profile obtained from the present study and the analytical results of Jha and Aina [7] are plotted in Figure 2(a) when the Knudsen number is equal to zero $(K n=$ $0)$. In this comparison, the inner and outer cylinders are stationary and the suction takes place from their walls. Furthermore, the mixed convective parameter and Prandtl number are considered 50 and 7 , respectively. According to Figure 2(b), by considering the 


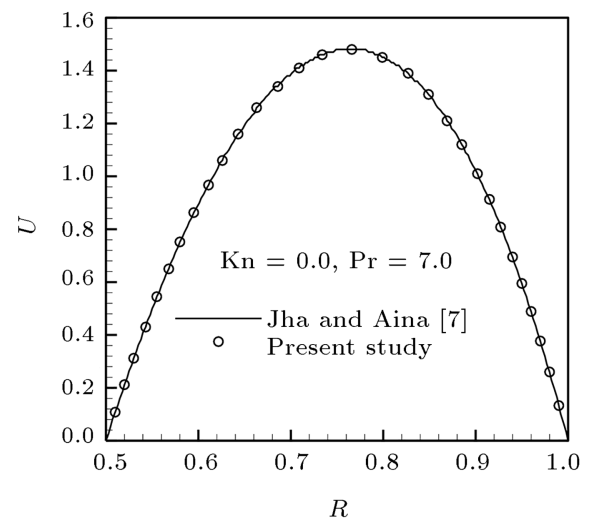

(a)

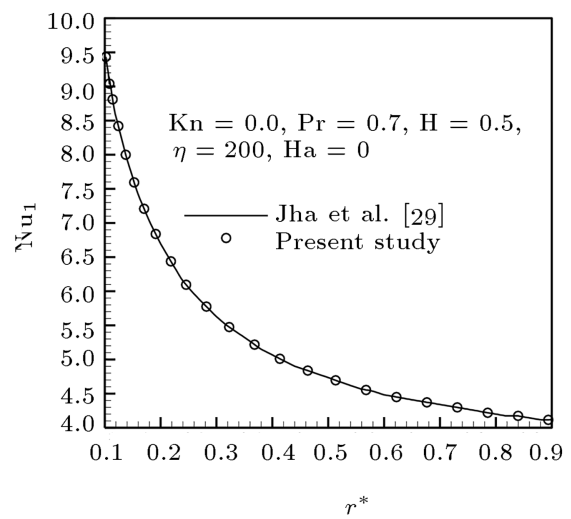

(b)

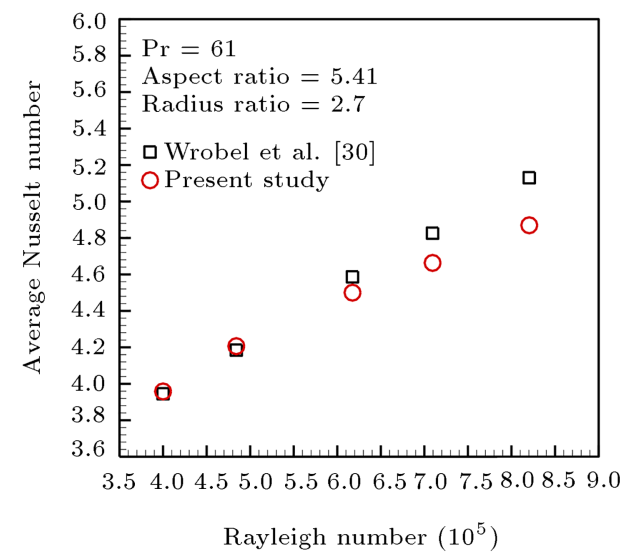

(c)

Figure 2. Validation: (a) The comparison of velocity profile in the annulus with Jha and Aina's [7] work in $\eta=50$, and $S=-0.5$, (b) the Nusselt number on inner cylinder of annulus compared with Ref. [29], and (c) Comparison of results of average Nusselt number in terms of Rayleigh number with Ref. [30].

suction/injection, the Nusselt number values achieved on the inner cylinder wall in different radius ratios have been compared with the results of Jha et al. [29]. As can be seen, the results in Figure 2(a) and (b) overlap one another completely. In Figure 2(c), a comparison has been carried out between the result of the present study and the experimental results of Wrobel et al. [30]. In this figure, the average Nusselt number is plotted in terms of Rayleigh number in a vertical annulus for conditions where the horizontal walls are considered insulated and the vertical walls have a constant temperature. As observed, there is a relatively close correspondence between both results.

\section{Results and discussions}

Generally, finding the exact solution for Navier-Stokes equations has many mathematical complexities. However, Eqs. (8) and (9) render exact solutions for this problem. It is worth mentioning that the exact solution results of the steady state case of the present problem, when the cylinder walls move in axial directions at constant velocity, are presented in references [23,31]. As mentioned, this study aims at investigating a situation in which the cylinder wall moves with timedependent velocity in a transient state. There are two categories of answers for Eqs. (8) and (9). If $S= \pm \frac{1}{\operatorname{Pr}}$, the energy equation turns into the classical heat equation with known results for $\theta(R, \theta)$ which can be replaced into the momentum equation to obtain the velocity distribution. The second situation is when $S \neq \pm \frac{1}{\mathrm{Pr}}$ where numerical methods are used to attain the desired results for $\theta(R, \tau)$ and which are then used in momentum equations.

\subsection{Temperature variation analysis}

The diagrams and contours of dimensionless temperature variations in the distance between the inner and outer cylinders for two states of $S= \pm 2$ at different times are illustrated in Figure 3. The inner cylinder has a higher temperature than the fluid and, therefore, heat transfer occurs due to the temperature difference. As observed, when the fluid is suctioned from the annulus axis to the outer cylinder $(S=-2)$, the fluid tempera- 


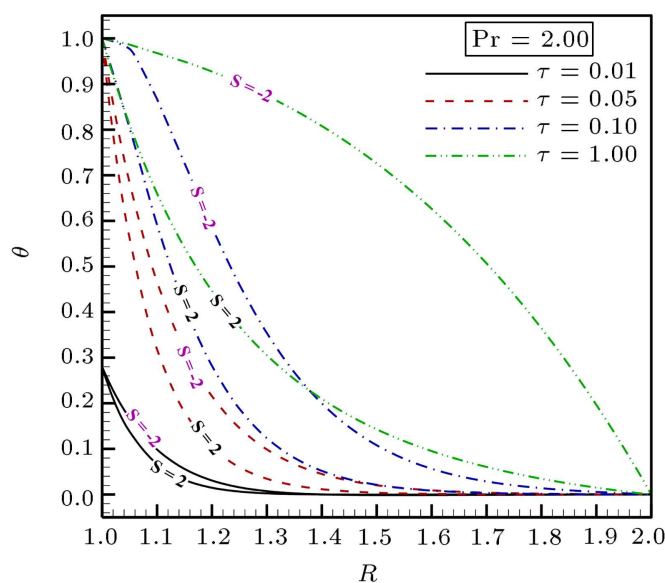

$\theta$

0.959596

0.909091

0.858586

0.808081

0.757576

0.707071

0.656566

0.606061

0.555556

0.505051

0.454545

0.40404

0.353535

0.30303

0.252525

0.20202

0.151515

0.10101

0.0505051

0

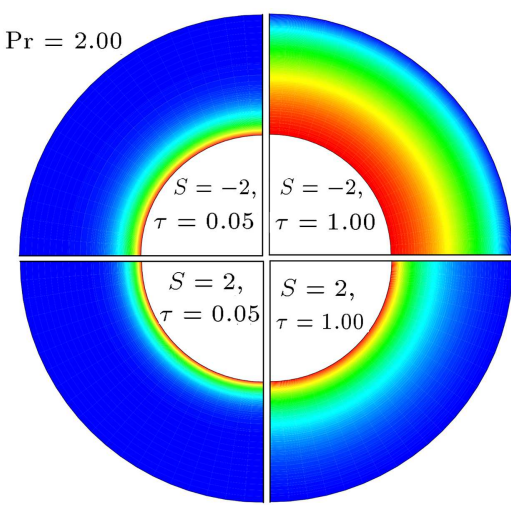

(a)
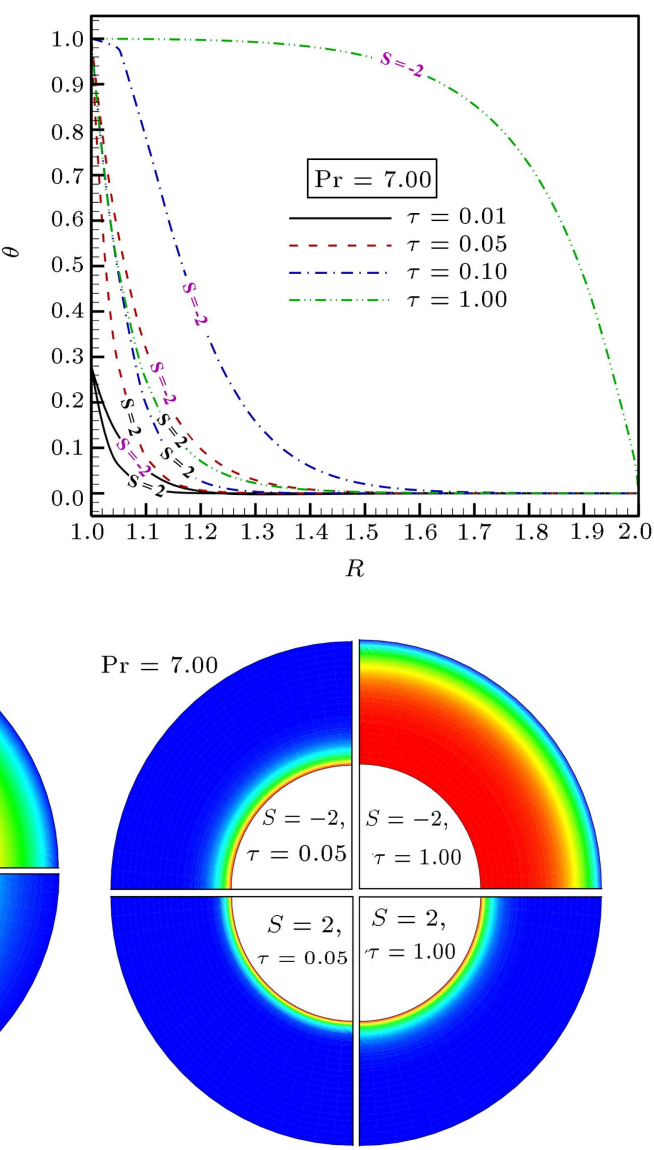

(b)

Figure 3. Diagrams and contours of dimensionless temperature in the distance between two cylinders at different times in $S= \pm 2, \lambda=2$, and $\eta=5:$ (a) $\operatorname{Pr}=2.00$ and (b) $\operatorname{Pr}=7.00$.

ture field penetrates into the outer wall direction, and the fluid temperature increases in the distance between the two cylinders as time increases. Furthermore, when the fluid is injected into the cylinder axis direction $(S=2)$, the temperature increases near the inner cylinder as time enhances. It should be noted that the obtained results indicate the in effectiveness of cylinder wall motion on temperature profile. Prandtl number is a dimensionless number that is obtained from the ratio of momentum diffusivity to thermal diffusivity. In Figure 3(a) and (b), the diagrams and contours of the dimensionless temperature are compared for the two Prandtl numbers of 2 and 7 . As can be observed, the thickness of the thermal boundary layer decreases and the slope of the dimensionless temperature diagram increases by increasing the Prandtl number.

\subsection{Fluid flow analysis}

Variations of dimensionless velocity profile and contour in the distance between the inner and outer cylinders at different times and for two different rates of suction/injection $S= \pm 2$ are displayed in Figure 4 . The movement of the inner cylinder wall is time- dependent in the axial direction and corresponds to an exponential motion of $F(\tau)=e^{-\tau}$. As can be seen, the movement of the inner cylinder wall affects the fluid velocity profile. With regard to the fact that the selected velocity profile is of a slowing down type, the velocity heightens initially as time increases and then it reduces. When the fluid is suctioned from the direction of the annulus axis toward its external part $(S=-2)$, the fluid is drawn into the outer cylinder wall and the velocity profile takes a parabolic form. This process causes the velocity to increase in the middle area between the two cylindrical pipes. Moreover, as the fluid is injected toward the annulus axis, the volume of fluid increases near the inner cylinder wall.

In Figure 5, the effect of the changes of dimensionless mixed convective parameters on the dimensionless velocity profile of the fluid and its contour between two inner and outer cylinders is depicted at different times. The outer cylinder wall is stationary and the inner cylinder moves in the axial direction exponentially and slows down according to the time-dependent function of $F(\tau)=e^{-\tau}$. As in Eq. (5), the mixed convective parameter is a dimensionless number and is defined 

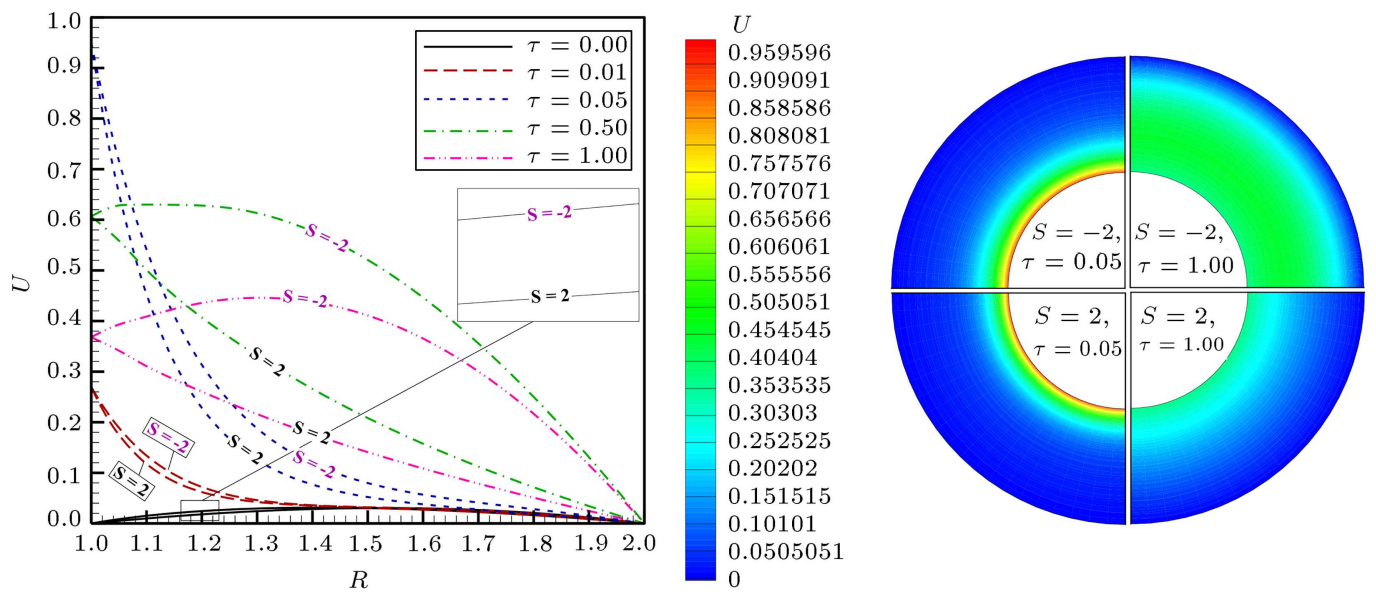

Figure 4. Diagram and contour of dimensionless velocity in the distance between the two cylinders at different times in $S= \pm 2, \operatorname{Pr}=7.0, \lambda=2, \eta=5$, and $F(\tau)=e^{-\tau}$.
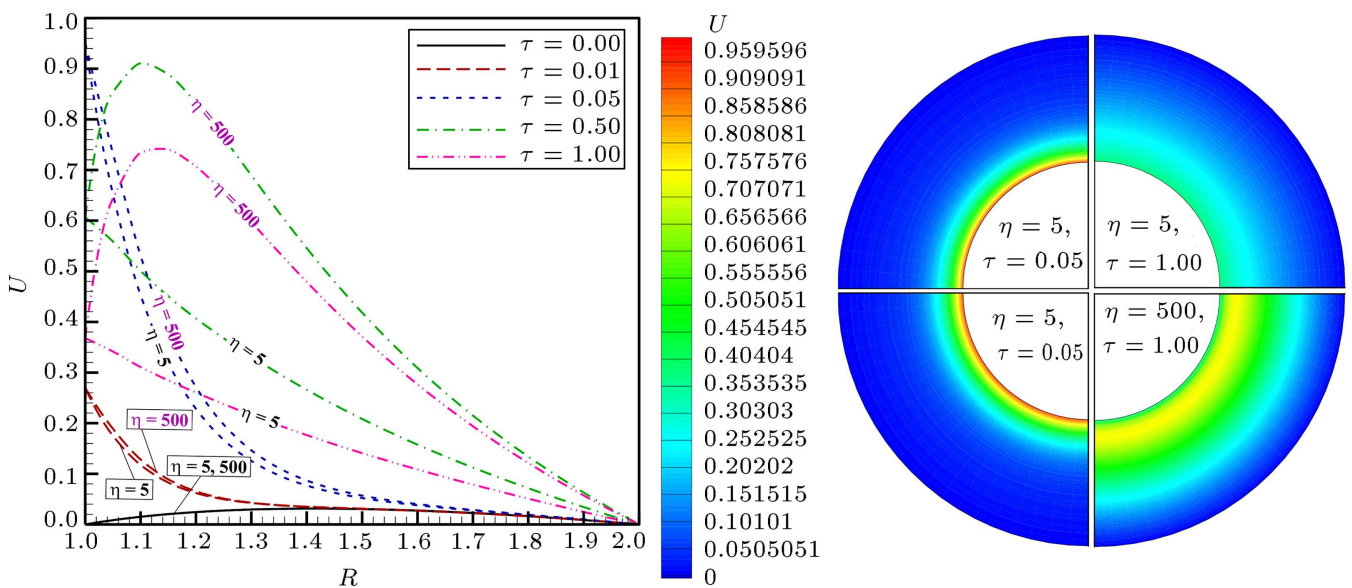

Figure 5. Diagram and contour of dimensionless velocity in the distance between two pipes at different times $\eta=5,500$, $\operatorname{Pr}=7.0, \lambda=2, S=2$, and $F(\tau)=e^{-\tau}$.

as the ratio of Grashof number to Reynolds number. Since the inner cylinder wall has a higher temperature, the natural convection term becomes stronger for larger mixed convective parameters and, thus, causes the fluid velocity to increase near the hot wall (of the inner cylinder). Furthermore, the velocity accelerates initially near the inner cylinder wall as time increases and the velocity function reduces again because of being slowed down. A key point to note in Figure 5 is that when $\eta$ equals 500, the influence of the fluid velocity field on the range between two cylinders enhances owing to the increase of buoyancy force, and it creates a parabolic profile between the two cylinders.

As mentioned earlier, various functions with different application modes, such as slowing down exponent $\left(F(\tau)=e^{-\tau}\right)$, slowing down fraction $(F(\tau)=$ $1 / \tau)$ and periodic trigonometry $(F(\tau)=\sin \tau)$, are employed to study the effect of wall motion on the flow and heat transfer of the fluid in the space between two cylinders. In Figure 6, the changes of fluid flow pattern caused by the movement of the inner cylinder wall in the axial direction are presented by employing each of these functions. With regard to Figure 6(a) and (b), the fluid velocity heightens on the wall of the inner cylinder as time increases and then it declines and reaches the damping state. As can be observed, the amount of velocity augmentation is abrupt and considerable in the state of $(F(\tau)=1 / \tau)$.

Moreover, according to Figure $6(\mathrm{c})$, the fluid velocity near the inner cylinder wall is periodically increased and decreased by using the trigonometric function. In Figure 6(d), a comparison is made between the three employed functions simultaneously. Clearly, by comparing velocity profiles at the time of $\tau=1$, functions $F_{2}, F_{3}$ and $F_{1}$ apply the maximum velocity to the fluid adjacent to the wall, respectively. As a consequence, more velocity changes are applied to the fluid adjacent to the inner cylinder wall by employing the $F_{2}$ function. In addition, as the dimensionless time increases to $\tau=3$, the wall velocity decreases and the fluid velocity also slows down. It is evident that the functions $F_{1}, F_{2}$ and $F_{3}$ have the highest velocity 


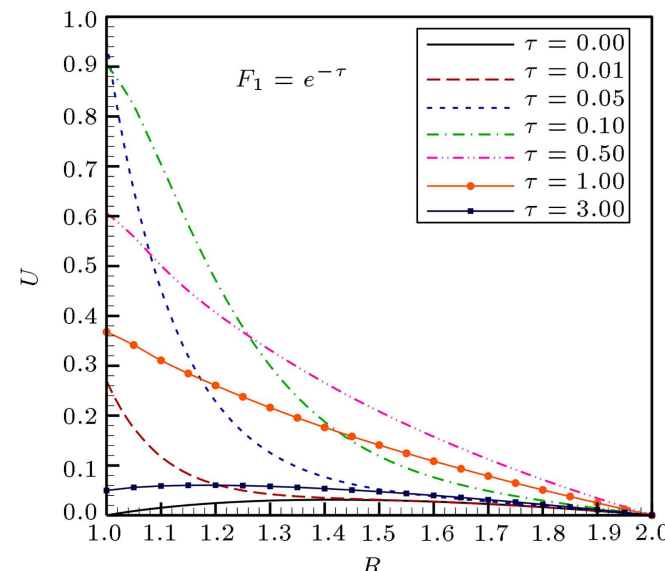

(a)

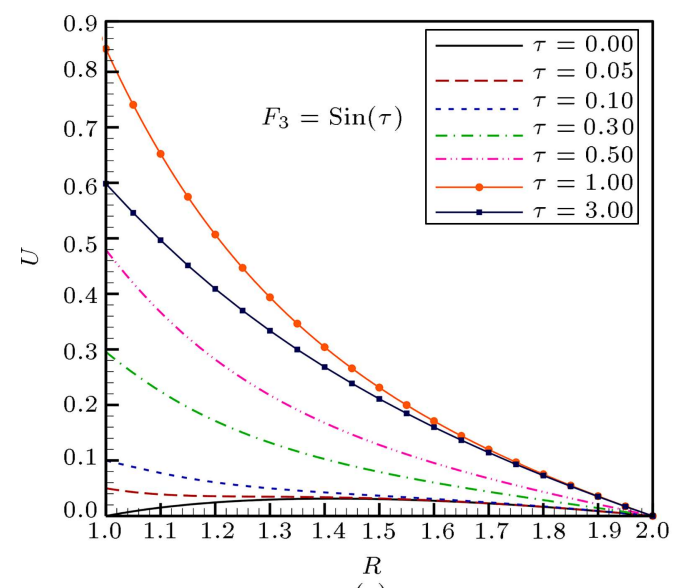

(c)

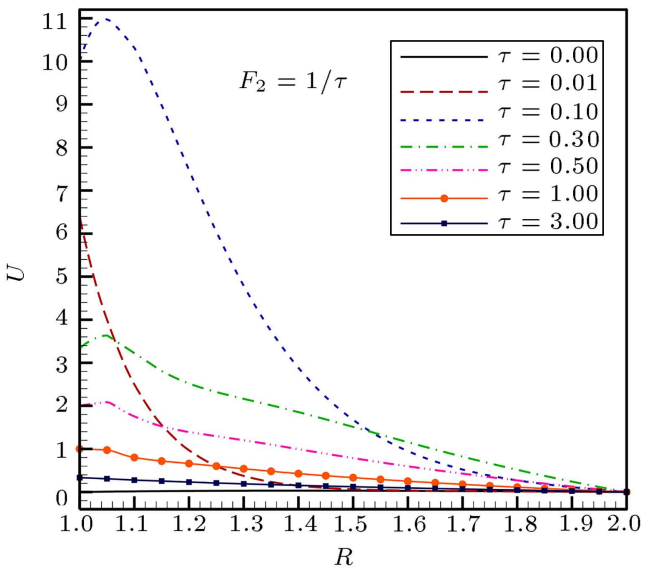

(b)

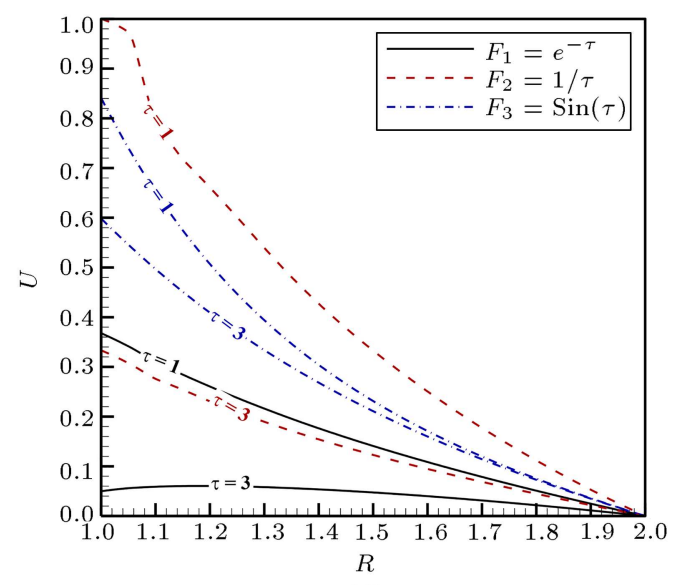

(d)

Figure 6. Diagram of dimensionless velocity in the distance between two cylinders for the case that the inner cylinder wall moves with time-dependent velocity: (a) $F_{1}=e^{-\tau}$, (b) $F_{2}=\frac{1}{\tau}$, (c) $F_{3}=\operatorname{Sin}(\tau)$, and (d) Comparison in $\operatorname{Pr}=7.0$, $\lambda=2, \eta=5, S=2$.

reduction, respectively, at the dimensionless time of $\tau=3$. Accordingly, function $F_{1}$ reaches the steady state sooner than the other functions.

\subsection{Heat transfer analysis}

The Nusselt number is a dimensionless parameter used to measure the amount of heat transfer at the boundaries of a system. According to Eq. (11), the Nusselt number is defined as the ratio of convection heat transfer rate to the conduction heat transfer rate. In Figure 7, the Nusselt number is plotted on the walls of inner and outer cylinders at different times when the inner cylinder wall moves with various time-dependent functions. According to Figure 7, the highest Nusselt numbers on the inner and outer walls are related to the functions of $F(\tau)=1 / \tau$ and $F(\tau)=e^{-\tau}$, respectively. Besides, the rate of Nusselt number change on both walls is incremental with increasing time, so that the enhancement of Nusselt number on the inner cylinder wall is noticeable during early times.

In Figure 8, the effect of mixed convection parameter changes on Nusselt number is presented at different

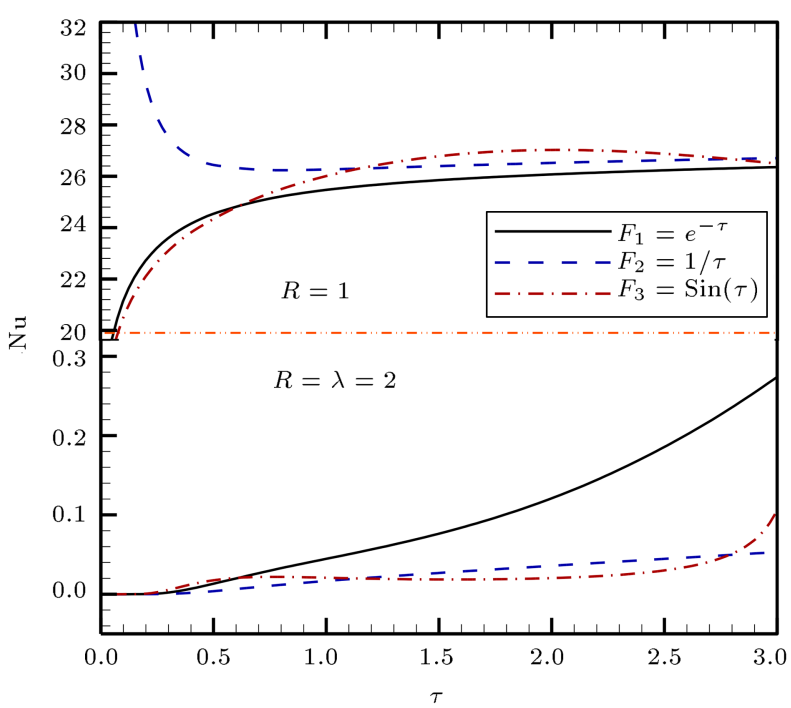

Figure 7. Diagram of Nusselt number on the walls of inner and outer cylinders in terms of dimensionless time in three different states of $F_{1}=e^{-\tau}, F_{2}=\frac{1}{\tau}$, and $F_{3}=\operatorname{Sin}(\tau)$ as a time dependent function of inner cylinder wall motion in $\operatorname{Pr}=7.0, \lambda=2, \eta=5$, and $S=2$. 

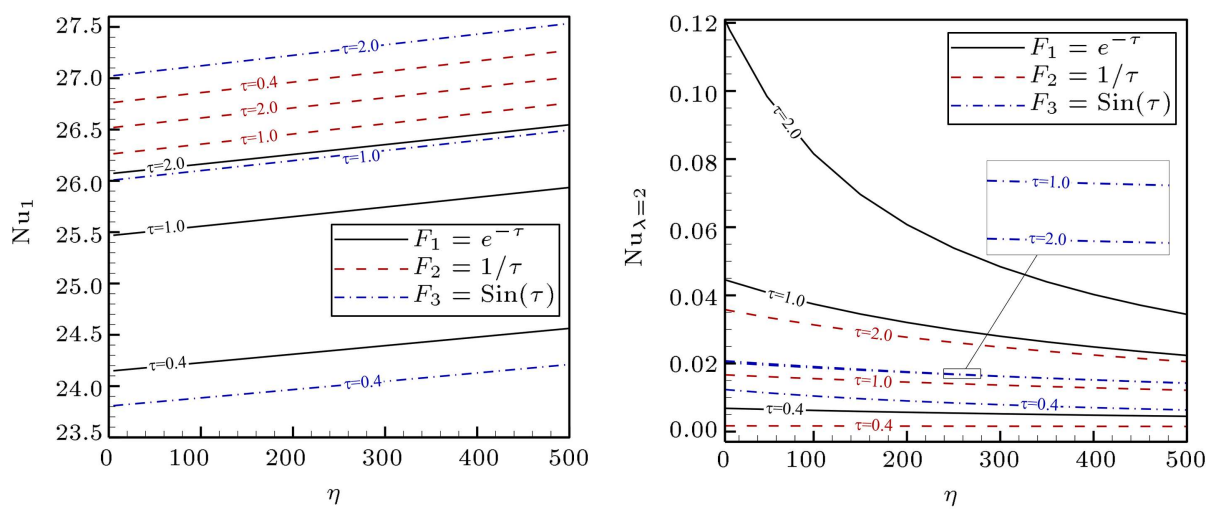

Figure 8. Diagram of Nusselt number in terms of mixed convection parameter: (a) On the wall of inner cylinder $(R=1)$ and (b) on the wall of inner cylinder $(R=\lambda=2)$ in three different states of $F_{1}=e^{-\tau}, F_{2}=\frac{1}{\tau}$, and $F_{3}=\operatorname{Sin}(\tau)$ as a time-dependent function of the inner cylinder wall motion in $\operatorname{Pr}=7.0$ and $S=2$.

times for three different states of wall motion with time dependent velocity. As can be seen, the buoyancy force of the fluid increases near the hot wall (of the inner cylinder) with the augmentation of $\eta$ and, consequently, the Nusselt number increases on the wall of the inner cylinder and decreases on the wall of the outer cylinder. By comparing the different states of the inner cylinder wall motion, it is found that, for example, at the time interval of $(\tau=2.00)$, the highest Nusselt numbers on the inner and outer cylinder walls are related to the functions of $F(\tau)=\sin \tau$ and $F(\tau)=e^{-\tau}$, respectively.

\subsection{Surface tension analysis}

The variations of dimensionless shear stress on the walls of inner and outer cylinders in terms of time for different states of wall motion with various functions are shown in Figure 9. It is apparent that the highest shear stress on the inner cylinder wall is related to the function of $F(\tau)=1 / \tau$, whose amount is noticeable. According to Figure 6(b), since shear stress corresponds to the rate of velocity variations, the sharp increase of shear stress for this function can be attributed to the growth of the velocity gradient near the inner cylinder wall. Moreover, the process of shear stress changes, in terms of time, is different, depending on the type of function used. For instance, when the trigonometric function of $F(\tau)=\sin \tau$ is utilized, its shear stress diagram is also in periodic format. According to the diagram, it is clear that where the velocity gradient has negative values, the shear stress also shows negative values.

The effects of $S$ parameter changes on shear stress on the walls of the inner and outer cylinders for different functions applied to the inner cylinder wall are illustrated in Figure 10. As can be seen, regardless of the type of function used, the shear stress decreases and increases, respectively, on the walls of the inner and outer cylinders by enhancing $S$ from negative to positive values. For example, by injecting

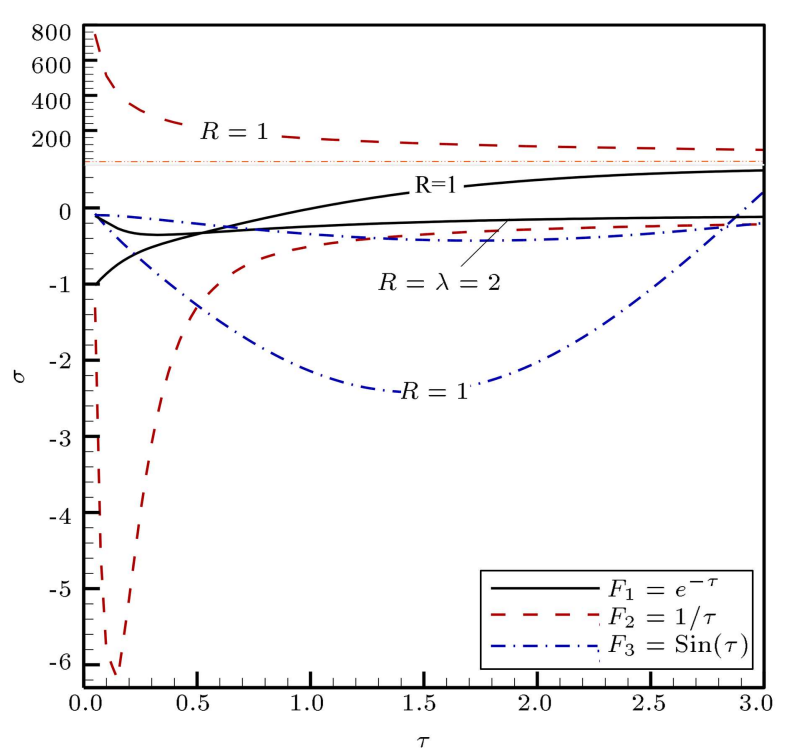

Figure 9. Diagram of dimensionless shear stress in terms of time on the walls of inner and outer cylinders in three different states of $F_{1}=e^{-\tau}, F_{2}=\frac{1}{\tau}$, and $F_{3}=\operatorname{Sin}(\tau)$ as a time dependent function of inner cylinder wall motion in $\operatorname{Pr}=7.0, \lambda=2, \eta=5$, and $S=2$.

the fluid toward the annulus axis, the velocity gradient diminishes near the inner cylinder wall and causes the shear stress to reduce on the inner cylinder wall. The opposite of this process occurs for the shear stress on the outer cylinder. As already mentioned, when the function of $F(\tau)=1 / \tau$ is utilized, the amount of shear stress is greater on the wall of the inner cylinder than the other situations, due to the drastic changes of the velocity gradient.

\section{Conclusions}

In this study, the role of movement of walls with timedependent velocity on flow and mixed convection in a vertical cylindrical annulus with suction/injection has 


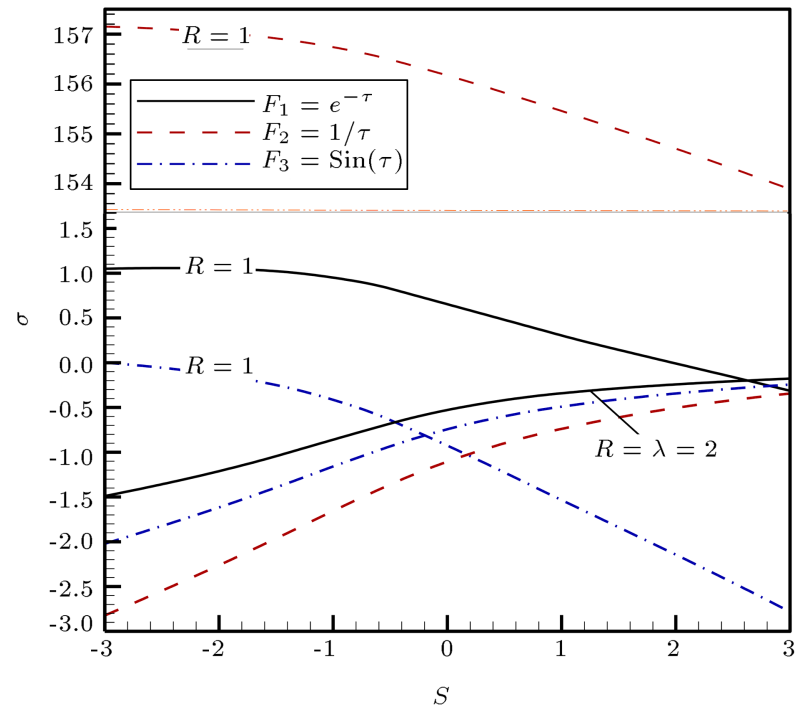

Figure 10. Diagram of dimensionless shear stress based on suction/injection parameter on the walls of inner and outer cylinders in three different states of $F_{1}=e^{-\tau}$, $F_{2}=\frac{1}{\tau}$, and $F_{3}=\operatorname{Sin}(\tau)$ as a time dependent function of inner cylinder wall motion in $\tau=1.0, \operatorname{Pr}=7.0, \lambda=2$, and $\eta=5$.

been considered. An exact solution of the NavierStokes and energy equations has been obtained. It has been shown that the energy equation turns to a heat equation in the case of a transpiration function equal to the inverse of the Prandtl number. Otherwise, the behavior of the flow and heat transfer of the fluid has been investigated by considering selected types of timedependent functions for the movement of the cylinder walls, as slowing down exponent $F(\tau)=e^{-\tau}$, slowing down fraction $F(\tau)=1 / \tau$ and periodic trigonometry $F(\tau)=\sin \tau$. The walls of inner and outer cylinders are perforated and the effects of suction/injection on these walls have also been studied. The obtained results have revealed that:

- The movement of cylinder walls has no effect on the dimensionless temperature profile;

- The comparison of three time-dependent motion functions indicated that the fluid reaches the steady state earlier than the other states when the function of $F(\tau)=e^{-\tau}$ is used in the inner cylinder wall. Furthermore, the maximum Nusselt number is obtained on the outer wall through using this function;

- The comparison of time-dependent functions shed light on the fact that the function of $F(\tau)=1 / \tau$ causes greater velocity changes than the other functions. This function also brings about a significant increase of the fluid velocity near the inner cylinder wall. Also, the maximum Nusselt number and shear stress are created on the inner cylinder wall by using this function;
- The rate of Nusselt number changes is incremental on the wall of both cylinders;

- With the enhancement of $\eta$, Nusselt number increases and decreases, respectively, on the inner and outer walls;

- Regardless of the type of the function used, the shear stress decreases and increases, respectively, on the inner and outer cylinder walls by enhancing the suction/injection rate from negative to positive values.

\section{Nomenclature}

$b$

$C_{p}$

$D_{h}$

$\mathrm{Gr}$

$g$

$k$

$\mathrm{Nu}$

$p$

Pr Prandtl number

$r \quad$ Axis in the cylindrical coordinates

$R \quad$ Dimensionless radios

Re Reynolds number

$S \quad$ Suction/injection parameter

$t \quad$ Time (S)

$T \quad$ Temperature of fluid (K)

$T_{0} \quad$ Wall temperature of outer cylinder (K)

$T_{1} \quad$ Wall temperature of inner cylinder $(\mathrm{K})$

$\Delta T \quad$ Temperature difference, $\Delta T=T_{1}-T_{0}$

$u \quad$ Velocity in $\mathrm{z}$ direction $(\mathrm{m} / \mathrm{s})$

$u_{0} \quad$ Reference constant velocity $\left(\mathrm{m}^{2} / \mathrm{s}\right)$

$U \quad$ Dimensionless velocity

$z \quad$ Axis in the cylindrical coordinates

\section{Greek letters}

$\alpha_{T} \quad$ Thermal diffusivity $\left(\mathrm{m}^{2} / \mathrm{s}\right), \alpha_{T}=$ $k / \rho C_{p}$

$\beta \quad$ Thermal expansion coefficient $(1 / \mathrm{K})$

$\eta \quad$ Mixed convection parameter

$\theta \quad$ Dimensionless temperature of fluid

$\lambda$ Ratio of the radius between two cylinders

$\mu \quad$ Dynamic viscosity $(\mathrm{kg} /(\mathrm{m} . \mathrm{s}))$

$\rho \quad$ Density $\left(\mathrm{kg} / \mathrm{m}^{3}\right)$

$\nu \quad$ Kinematic viscosity $\left(\mathrm{m}^{2} / \mathrm{s}\right), \nu=\mu / \rho$ 
Dimensionless time

$\sigma$

Dimensionless shear stress

\section{Subscripts}

$l \quad$ Value on inner wall

$b \quad$ Bulk temperature

$\lambda \quad$ Value on outer wall

\section{References}

1. Wu, T.-H., Xu, Z., and Jackson, J. "Mixed convection heat transfer to water flowing through a vertical passage of annular cross section: part 2", Chemical Engineering Research and Design, 80(3), pp. 246-251 (2002).

2. Joshi, H.M. "Fully developed natural convection in an isothermal vertical annular duct", International Communications in Heat and Mass Transfer, 14(6), pp. 657-664 (1987).

3. Chen, S., Tölke, J., and Krafczyk, M. "Numerical investigation of double-diffusive (natural) convection in vertical annuluses with opposing temperature and concentration gradients", International Journal of Heat and Fluid Flow, 31(2), pp. 217-226 (2010).

4. Iannello, V., Suh, K.Y., and Todreas, N.E. "Mixed convection friction factors and Nusselt numbers in vertical annular and subchannel geometries", International Journal of Heat and Mass Transfer, 31(10), pp. 2175-2189 (1988).

5. Malvandi, A., Moshizi, S., Soltani, E.G., and Ganji, D. "Modified Buongiorno's model for fully developed mixed convection flow of nanofluids in a vertical annular pipe", Computers \& Fluids, 89, pp. 124-132 (2014).

6. Avcı, M. and Aydı, O. "Mixed convection in a vertical microannulus between two concentric microtubes", Journal of Heat Transfer, 131 (1), p. 014502 (2009).

7. Jha, B.K. and Aina, B. "Mathematical modelling and exact solution of steady fully developed mixed convection flow in a vertical micro-porous-annulus", Afrika Matematika, 26(7-8), pp. 1199-1213 (2015).

8. Jha, B.K., Aina, B., and Muhammad, S. "Combined effects of suction/injection and wall surface curvature on natural convection flow in a vertical micro-porous annulus", Thermophysics and Aeromechanics, 22(2), pp. $217-228$ (2015).

9. Husain, S. and Siddiqui, M.A. "Experimental and numerical analysis of transient natural convection of water in a high aspect ratio narrow vertical annulus", Progress in Nuclear Energy, 106, pp. 1-10 (2018).

10. Husain, S., Siddiqui, M.A., and Khan, S.A. "Effect of geometrical parameters on natural convection of water in a narrow annulus", Progress in Nuclear Energy, 112, pp. 146-161 (2019).
11. Husain, S. and Siddiqui, M.A. "Experimental and numerical analyses of natural convection flow in a partially heated vertical annulus", Numerical Heat Transfer, Part A: Applications, 70(7), pp. 763-775 (2016).

12. Husain, S. and Siddiqui, M.A. "Numerical and experimental analysis of natural convection flow boiling of water in internally heated vertical annulus", Numerical Heat Transfer, Part A: Applications, 73(9), pp. 624653 (2018).

13. Mustafa, J., Husain, S., and Siddiqui, M.A.J.E.H.T. "Experimental studies on natural convection of water in a closed-loop vertical annulus", Experimental Heat Transfer an International Journal, 30(1), pp. 25-45 (2017).

14. Huang, S. and Chun, C.H. "A numerical study of turbulent flow and conjugate heat transfer in concentric annuli with moving inner rod", International Journal of Heat and Mass Transfer, 46(19), pp. 3707-3716 (2003).

15. Lin, S. and Hsieh, D. "Heat transfer to generalized Couette flow of a non-Newtonian fluid in annuli with moving inner cylinder", Journal of Heat Transfer, 102(4), pp. 786-789 (1980).

16. Strafford, K.N., Datta, P.K., and Coogan, C., Coatings and Surface Treatment for Corrosion and Wear Resistance, Published for Institution of Corrosion Science and Technology by Ellis Horwood, Market Cross House, Cooper Street, Chichester, West Sussex PO 19 1 EB, England, 1984. 362 (1984).

17. Shigechi, T. and Lee, Y. "An analysis on fully developed laminar fluid flow and heat transfer in concentric annuli with moving cores", International Journal of Heat and Mass Transfer, 34(10), pp. 2593-2601 (1991).

18. Chamkha, A.J. "Unsteady MHD convective heat and mass transfer past a semi-infinite vertical permeable moving plate with heat absorption", International Journal of Engineering Science, 42(2), pp. 217-230 (2004).

19. Abedini, A. and Rahimi, A.B. "Numerical study of mixed convection in an annulus between concentric rotating cylinders with time-dependent angular velocity", Iranian Journal of Science and TechnologyTransactions of Mechanical Engineering, 36, pp. 165180 (2012).

20. Saleh, R. and Rahimi, A.B. "Axisymmetric stagnationpoint flow and heat transfer of a viscous fluid on a moving cylinder with time-dependent axial velocity and uniform transpiration", Journal of Fluids Engineering, 126(6), pp. 997-1005 (2004).

21. Jha, B., Joseph, S., and Ajibade, A. "Transient freeconvective flow through a vertical porous annulus", Proceedings of the Institution of Mechanical Engineers, Part E: Journal of Process Mechanical Engineering, 226(2), pp. 105-116 (2012). 
22. Jha, B., Samaila, A., Ajibade, A. "Unsteady/steady natural convection flow of reactive viscous fluid in a vertical annulus", International Journal of Applied Mechanics Engineering, 18(1), pp. 73-83 (2013).

23. Shakiba, A. and Rahimi, A.B. "Mixed convective flow of electrically conducting fluid in a vertical cylindrical annulus with moving walls adjacent to a radial magnetic field along with transpiration", Journal of Fluids Engineering, 141, pp. 1-11 (2019).

24. Busedra, A. and Tavoularis, S. "Numerical simulations of natural convective heat transfer in vertical concentric and eccentric annular channels", Journal of Heat Transfer, 140(10), p. 102502 (2018).

25. Hekmat, M.H. and Ziarati, K.K. "Effects of nanoparticles volume fraction and magnetic field gradient on the mixed convection of a ferrofluid in the annulus between vertical concentric cylinders", Applied Thermal Engineering, 152, pp. 844-857 (2019).

26. Jha, B. and Oni, M. "Natural convection flow in a vertical annulus with time-periodic thermal boundary conditions", Propulsion Power Research, 8(1), pp. 4755 (2019).

27. Jha, B.K. and Malgwi, P.B. "Effects of Hall current and magnetic field inclination on hydromagnetic natural convection flow in a micro-channel with asymmetric thermal boundary condition", Journal of Thermal Science Engineering Applications, 12(3) (2020).

28. Abbas, Z., Mehdi, I., Hasnain, J., and Aly, S. "Role of suction/injection on natural convection flow of magnetite $\mathrm{Fe}_{3} \mathrm{O}_{4}$ nanoparticles in vertical porous micro-annulus between two concentric tubes: A purely analytical approach", Arabian Journal for Science, 44(9), pp. 8113-8122 (2019).

29. Jha, B.K., Oni, M.O., and Aina, B. "Steady fully developed mixed convection flow in a vertical micro- concentric-annulus with heat generating/absorbing fluid: an exact solution", Ain Shams Engineering Journal, 9(4), pp. 1289-1301 (2018).

30. Wrobel, W., Fornalik-Wajs, E., and Szmyd, J.S. "Experimental and numerical analysis of thermo-magnetic convection in a vertical annular enclosure", International Journal of Heat Fluid Flow, 31(6), pp. 10191031 (2010).

31. Shakiba, A. and Rahimi, A.B. "Nanofluid flow and MHD mixed convection inside a vertical annulus with moving walls and transpiration considering the effect of Brownian motion and shape factor", Int. J. of Thermal Analysis and Calorimetry, 138, pp. 501-515 (2019).

\section{Biographies}

Ali Shakiba was born in Isfahan, Iran, in 1990. He received his BS degree in Mechanical Engineering from the Islamic Azad University, South Tehran Branch, Iran in 2012, and his MS degree in Mechanical Engineering from Mazandaran Institute of Technology, Iran in 2014. He is currently a PhD student of Mechanical Engineering in the Faculty of Mechanical Engineering at Ferdowsi University of Mashhad, Iran. His research interests include heat transfer, fluid mechanics, mathematics, nanofluid, MHD, FHD, CFD simulation and renewable energy.

Asghar Baradaran Rahimi was born in Mashhad, Iran, in 1951. He received his BS degree in Mechanical Engineering from Tehran Polytechnic, in 1974, and a PhD degree in Mechanical Engineering from the University of Akron, Ohio, USA, in 1986. He has been Professor in the Department of Mechanical Engineering at Ferdowsi University of Mashhad since 2001. His research and teaching interests include heat transfer and fluid dynamics, gas dynamics, continuum mechanics, applied mathematics and singular perturbation. 